\section{Passive RFID Strain-Sensor Based on Meander-Line Antennas}

\author{
C. Occhiuzzi, C. Paggi, and G. Marrocco
}

\begin{abstract}
The processing of backscattered signals coming from RFID tags is potentially useful to detect the physical state of the tagged object. It is here shown how to design a completely passive UHF RFID sensor for strain monitoring starting from a flexible meander-line dipole whose shape factor and feed section are engineered to achieve the desired sensing resolution and dynamic range. This class of devices is low-cost, promises sub-millimeter resolution and may found interesting applications in the Structural Health Monitoring of damaged structures and vehicles as well as during extreme and adverse events.
\end{abstract}

Index Terms-Passive sensor, sensor RFID tag, strain gauge, strain measurement.

\section{INTRODUCTION}

Processing the backscattered signals emitted by tags for Radio Frequency Identification (RFID) is a promising means to achieve passive low-cost sensing of things. Several examples have been proposed in the very last years [1], [2], demonstrating the use of RFID tags as "sensor-less" sensing devices with fully controllable response.

One of the most interesting parameters to be detected is the strain experimented by an object or a surface, with possible application to the Structural Health Monitoring (SHM) of buildings and vehicles. The damage detection has a severe need of devices able to continuously monitor possible anomalies in materials and/or in the geometric properties of a structural system, in both normal or extreme conditions, such as earthquakes, blast loading or cracks. An SHM system should be wireless, low-cost, fully autonomous and highly reliable [3].

The feasibility of using antennas in general as radio sensors for strain and crack monitoring has been investigated in [4]-[6]. An applied strain or a growing crack changes the size of the metallic patch, producing in turn a shift of the antenna's resonant frequency. However, all the proposed platforms require specific radar systems and dedicated receivers in order to detect such deformations.

RFID passive technology may instead offer a complete low-cost and off-the-shelf solution thanks to the availability of microchips transponder which digitally encode the signal transmitted back to the reader while preserving the analog electromagnetic interaction with the tagged item. Hence, when the object where the tag is attached on undergoes changes, the tag's electrical features accordingly modify, and these variations can be somehow detected remotely by the RFID reader which will be also able to discriminate the signals coming from a dense distribution of radio-sensors. A first example of strain-sensing tag was proposed in [7] where the antenna was printed over a stretchable substrate whose deformation produces a modification of the microstructure of the conductive ink and thus of the overall conductivity. However no attempt was done to control and increase the sensitivity during the antenna design.

Manuscript received March 14, 2011; revised May 22, 2011; accepted June 21, 2011. Date of publication August 22, 2011; date of current version December 02,2011 . This work was supported by the Italian Ministry of University and Research under Research Grant PRIN-2008 MULTITAG.

The authors are with the Dipartimento di Informatica Sistemi e Produzione, University of Roma Tor Vergata, Roma, RM 00133, Italy (e-mail: occhiuzzi@disp.uniroma2.it; marrocco@disp.uniroma2.it).

Digital Object Identifier 10.1109/TAP.2011.2165517

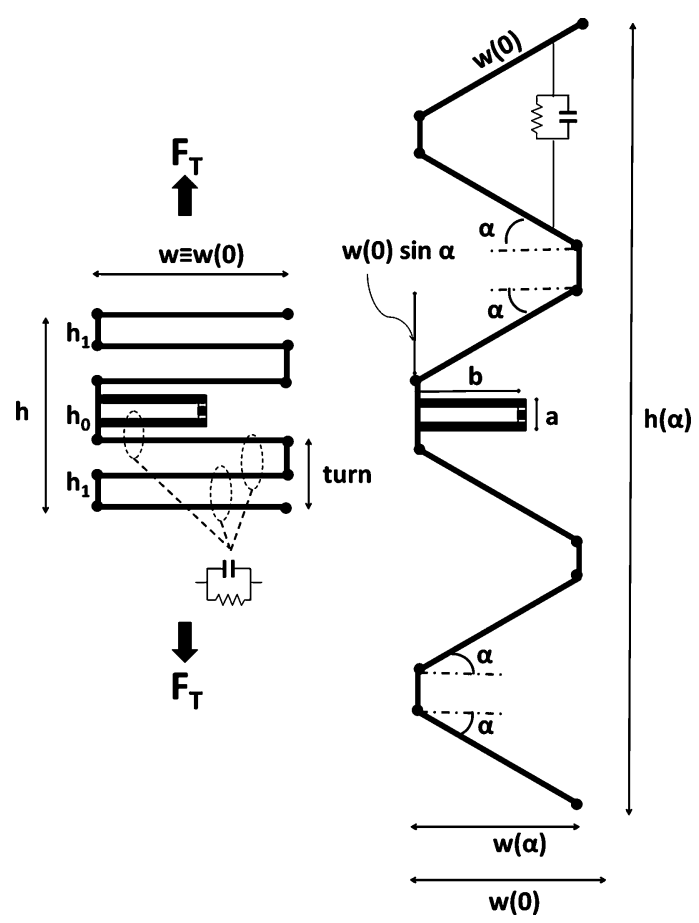

Fig. 1. Meander-line tag layout with $2 N=2$ turns (left) quiet state; (right) stretched state subjected to vertical tractive forces $F_{T}$.

A different and fully controllable layout is here proposed, for the UHF (868-956 MHz) RFID band, even if the same ideas may be naturally extended to higher frequencies, with improved resolution. The device is based onto a meander line antenna able to change its shape and consequently its radiation performances depending on the experienced deformation. A parametric analysis will show that it is possible to shape the sensitivity and the dynamic range of the RFID strain sensor by a proper choice of few geometrical parameters. The idea is finally demonstrated through a working prototype subjected to controlled stress.

\section{RATionale of StRain SENSING By UHF RFID}

The meander-line antenna (MLA) is a commonly used layout to obtain a large variety of RFID tags [8]. An example of symmetric MLA with $2 \mathrm{~N}$ foldings and a tuning T-match section is shown in Fig. 1 (left). The profile of the wire turns, useful to reduce the antenna size, strongly impacts on the input impedance, since the foldings may be seen as distributed capacitive loads of the structure depending in turn on the mutual distance between facing segments. The antenna gain is instead mainly imposed by the overall length $\left(h=h_{0}+2 \sum_{n=1}^{N} h_{n}\right)$ of the tag.

\section{A. Mechanical Model}

If the MLA is subjected to tensile stress through an applied axial force $\pm \mathbf{F}_{T}$, the antenna's shape factor changes (Fig. 1 (right)) as well. The distributed load, and hence both the input impedance and the antenna gain will be accordingly modified. More precisely, by assuming inextensible wires (rigid structure), the external forces will produce rotation of the folding elements by an angle $\alpha\left(F_{T}\right)$, consequently forcing the horizontal and the vertical translations of the moving nodes of the structure [9]. $w(\alpha)$ and $h(\alpha)$ here denote the overall size of the deformed MLA, being $w(0) \equiv w$ and $h(0) \equiv h$ the size of the MLA at rest. By neglecting the wire's radius, each turn of the MLA under stress 
produces an elongation equal to $3 w(0) \sin \alpha$ and hence the overall size of the MLA is simple expressed in terms of the structures at rest as

$$
\begin{aligned}
& w(\alpha)=w(0) \cos \alpha \\
& h(\alpha)=h(0)+6 N w(0) \sin \alpha
\end{aligned}
$$

and the overall elongation in the strain direction to be correlated with the force is hence $\Delta h=6 N w(0) \sin \alpha$.

\section{B. Electromagnetic Model}

During the RFID communication, the passive tag generates a backscattered modulation of the impinging interrogation carrier coming from the reader. The power collected by the reader [10] can be expressed by making explicit the dependence on the overall elongation $\Delta h$ :

$$
P_{R-T}[\Delta h]=\frac{1}{4 \pi}\left(\frac{\lambda_{0}}{4 \pi d^{2}}\right)^{2} P_{\text {in }} G_{R}^{2} \eta_{p}^{2} \operatorname{rcs}_{T}[\Delta h]
$$

where $r c s_{T}$ is the tag's radar cross-section [10],

$$
\operatorname{rcs}_{T}[\Delta h]=\frac{\lambda_{0}^{2}}{4 \pi} G_{T}^{2}[\Delta h]\left(\frac{2 R_{A}[\Delta h]}{\mid Z_{\bmod }+Z_{A}[\Delta h \mid}\right)^{2}
$$

where $d$ is the reader-tag distance, $G_{R}$ is the gain of the reader antenna, $G_{T}$ is the gain of the tag's antenna (as dependent on the deformation) when placed onto the tagged object. $P_{\text {in }}$ is the power entering in the reader antenna, $\eta_{p}$ is the polarization mismatch between the reader and the tag and finally $Z_{A}=R_{A}+j X_{A}$ and $Z_{\bmod }$ are the input impedance of the antenna and of the RFID microchip, respectively. It is worth recalling that the impedance $Z_{\bmod }$ is switched during the binary backscattering modulation between an open circuit $\left(r c s_{T}=0\right)$ and a finite value $Z_{\text {chip }}=R_{\text {chip }}+j X_{\text {chip }}$, (with $X_{\text {chip }}<0$ ).

The backscattered power $P_{R-T}$ is directly measurable by the reader in terms of the received signal strength indicator (RSSI), here assumed to correspond [10], [11] to the binary modulating state having $\left(Z_{\mathrm{mod}}=\right.$ $\left.Z_{\text {chip }}\right) . P_{R-T}$ carries information about the elongation $\Delta h$ of the tag thanks to the highlighted dependencies on impedance and gain. To be specific, when the reader-tag mutual position and $P_{\text {in }}$ are exactly reproduced in successive interrogations, it is possible to simply derive a data inversion (or calibration) curve $p_{s} \leftrightarrow \Delta h$, independently on the reader's gain, by referring the backscattered power to the rest condition $(\Delta h=0)$ of the tag

$$
\begin{aligned}
p_{S}[\Delta h] & \equiv \frac{P_{R-T}[\Delta h]}{P_{R-T}[0]} \\
& =\left(\frac{G_{T}[\Delta h]}{G_{T}[0]}\right)^{2} \frac{R_{A}[\Delta h]}{R_{A}[0]} \frac{\tau[\Delta h]}{\tau[0]}
\end{aligned}
$$

with $\tau[\Delta h]$ power transmission coefficient of the tag

$$
\tau[\Delta h]=\frac{4 R_{\text {chip }} R_{A}[\Delta h]}{\left|Z_{\text {chip }}+Z_{A}[\Delta h]\right|^{2}}
$$

which is maximum $(\tau \rightarrow 1)$ in case of complex matching $\left(Z_{A}(\Delta h)=\right.$ $Z_{\text {chip }}^{\star}$ ). The shape of data-inversion curve $p_{S}[\Delta h]$ is hence a combination of three effects produced by the change of the MLA, e.g., the variation of gain $G_{T}$, the variation of the impedance matching $(\tau)$ and finally the variation of the real part $R_{A}$ of the input impedance of the antenna. Other sensing indicators, based on he turn-on power or on the analog identifier, may be derived as described in [1], [12] and straightforwardly extended to the specific sensing problem.

\section{Electromagnetic Response to Mechanical Deformations}

An example about possible relationships among the geometric (read mechanical) parameters and the electromagnetic response of the MLA
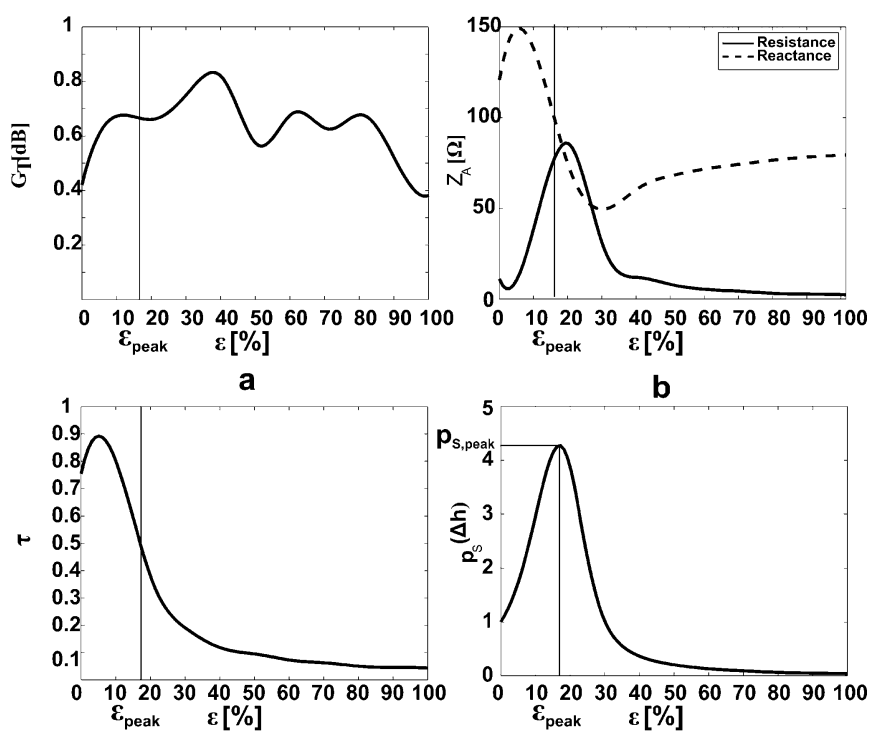

b

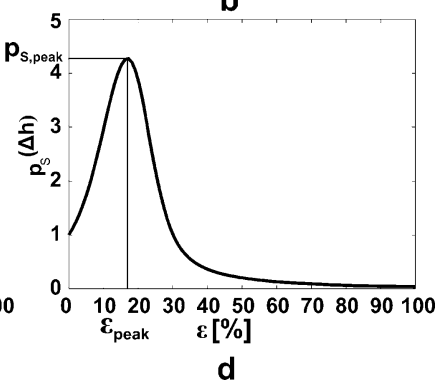

Fig. 2. Example of simulated gain (a), input impedance (b), power transmission coefficient (c) and backscattered power (d) of an $\mathrm{N}=1$ MLA tag (sizes in $[\mathrm{mm}]: h=36, h_{0}=6, h_{1}=7, w=36, a=4, b=18$, radius $r_{s}=0.5$ ) with respect to the strain $\epsilon$.

is shown in Fig. 2. A $3.6 \mathrm{~cm} \times 3.6 \mathrm{~cm}, N=1$, MLA is designed to have the best conjugate matching, in the rest position $\left(Z_{A}[0]=Z_{\text {chip }}^{*}\right)$, to the NXP-G2XM microchip with impedance $Z_{\text {chip }}=15-j 135 \Omega$. The antenna responses at $870 \mathrm{MHz}$ (the European RFID frequency) are predicted by a numerical model based on the finite-difference timedomain method [13].

Fig. 2 shows the simulated gain (a) and impedance (b) of the MLA under stress with respect to the strain $\epsilon$ [9] defined as

$$
\epsilon=\frac{\Delta h}{h} \cdot 100 \% \text {. }
$$

While the gain is only weakly sensitive to the deformation, both real and imaginary part of the tag's input impedance show instead significant variation according to a behavior similar to the frequency dependence of the input impedance of dipole. By increasing the strain $\epsilon$, the MLA's resistance $R_{A}$ initially increases as the square [14] of the antenna's overall height $h(\alpha)$ up to reach the anti-resonance condition, afterward giving rise to a non monotonic profile. The positive offset visible in the reactance is due to the inductive loading effect introduced by the T-match [15]. A similar Gaussian-like behavior can be found in the power transfer coefficient $\tau$ (Fig. 2(c)) which is indeed a function of the impedance. Finally, the resulting backscattered power $p_{S}$ (Fig. 2(d)) exhibits from (5), a sharp and non-monotonic dependence on the applied force with a peak value mainly imposed by the $R_{A}$ 's one (approximately seven times greater than the initial value). The initial positive slope, with high sensitivity, is then followed by a negative smoother profile, suggesting the possibility of several sensing options. Finally, beyond an $\epsilon=50 \%$ strain, the influence of the interactions between facing segments become negligible and the antenna performs as a straight dipole without possibility of further wireless sensing.

With the purpose to efficiently master the radio-sensor's response, the profile of the inversion curve is hereafter compressed into just two macroscopic features: the position and amplitude of the peak value, e.g., the $\left(\epsilon_{\text {peak }}, p_{s, \text { peak }}\right)$ couplet (see Fig. $\left.2(d)\right)$. It is easy to verify that a fully monotonic response with negative slope may be achieved if $\epsilon_{\text {peak }} \rightarrow 0$, while a monotonically increasing curve (positive slope) if $\epsilon_{\text {peak }} \gg 0$. It is finally expected that the slope of such curves may be shaped by acting on the amplitude $p_{s, \text { peak }}$. 


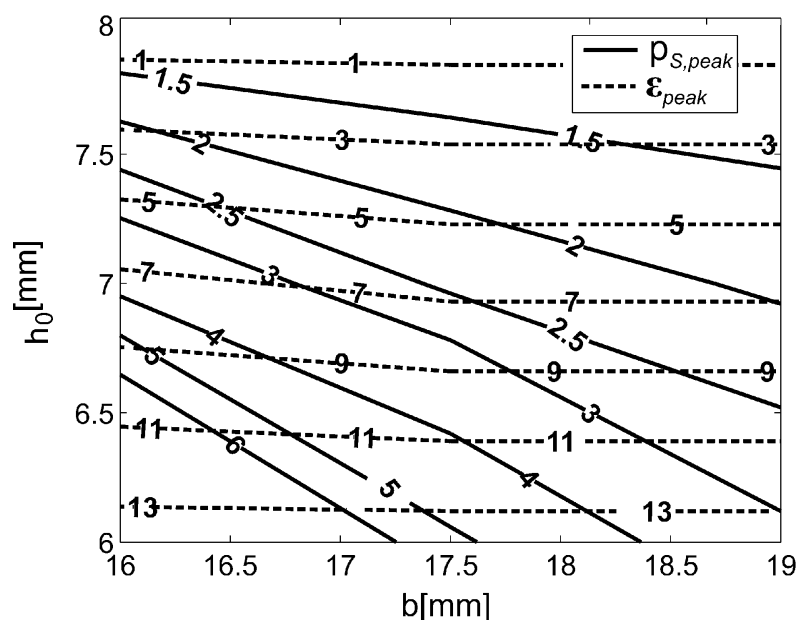

Fig. 3. Features of inversion curves (position $\epsilon_{\text {peak }}$ and peak $p_{S \text {,peak }}$ ) for the meander line tag in Fig. 1, with respect to the geometrical parameters $\left(b, h_{0}\right)$. The other sizes are fixed to the values in Fig. 2.

\section{Design Methodology}

The design of a strain radio-sensor must be strictly connected to the specific application it is going to monitor, and it is here finalized to shape the inversion curve. Some events show very slow and moderate dynamic range, requiring a monitoring system able to amplify minimal variations in long timescales. In other cases, especially during extreme events, the processes are characterized by rapid and large structural modifications. It is therefore required that in each specific process, or process' state, the inversion curve is able to properly follow the expected dynamics of $\epsilon$, being for example single-valued, e.g., monotonic, and steep.

The performances of the MLA-as-a-sensor, e.g., the maximum strain (or dynamic range) and the sensitivity can be derived by the macroscopic features $\left(\epsilon_{\text {peak }}, p_{s, \text { peak }}\right)$ introduced in the previous Section. The maximum strain $\epsilon_{\max }$ is defined for monotonic sensing curves having positive $\left(\epsilon_{\text {peak }}>0\right)$ and negative $\left(\epsilon_{\text {peak }}=0\right)$ slopes as

$$
\epsilon_{\max }= \begin{cases}\epsilon_{\text {peak }} & \epsilon_{\text {peak }}>0 \\ \epsilon_{0.1}: p_{s}\left(\epsilon_{0.1}\right)=0.1 p_{s}\left(\epsilon_{\text {peak }}\right) & \epsilon_{\text {peak }}=0\end{cases}
$$

wherein the 0.1 threshold (10\%) over the normalized backscattered power $p_{S}$ has been arbitrarily chosen in order that $p_{S}$ may be considered practically insensible to strain $\epsilon>\epsilon_{0.1}$ (see for instance Fig. 2(d)). The sensitivity of the sensor is defined as

$$
s[\%]=\frac{p_{s}\left(\epsilon_{\max }\right)-1}{\epsilon_{\max }}
$$

and gives the percentage variation (increase or decrease) of the collected backscattered power, with respect to the MLA's rest position, corresponding to $\epsilon_{\max }=1 \%$ of linear deformations.

The performance of the MLA sensor can be controlled by a proper choice of the geometrical size of the antenna layout. Although in a most general case the whole MLA's shape could be fully engineered to achieve a specific input impedance, as in [16], for the sake of simplicity the foldings are here considered fixed while the control parameters are just the length $h_{0}$ of the central segment where the T-match is connected, and the length $b$ of this last one. Fig. 3 gives the simulated chart of $\left(\epsilon_{\text {peak }}, p_{s, \text { peak }}\right)$ vs. the geometrical parameters $\left(h_{0}, b\right)$ and it is useful to design the tag for a required sensitivity and dynamic range. It is worth noticing that the position $\epsilon_{\text {peak }}$ of the backscattered power's peak (that indirectly imposes the dynamic range of the strain radio-sensor) is only controlled by the parameter $h_{0}$ since the iso-lines for $\epsilon_{\text {peak }}$ are almost horizontal. The change in $h_{0}$ in fact directly affects the electrical length of the MLA and hence the impedance curve

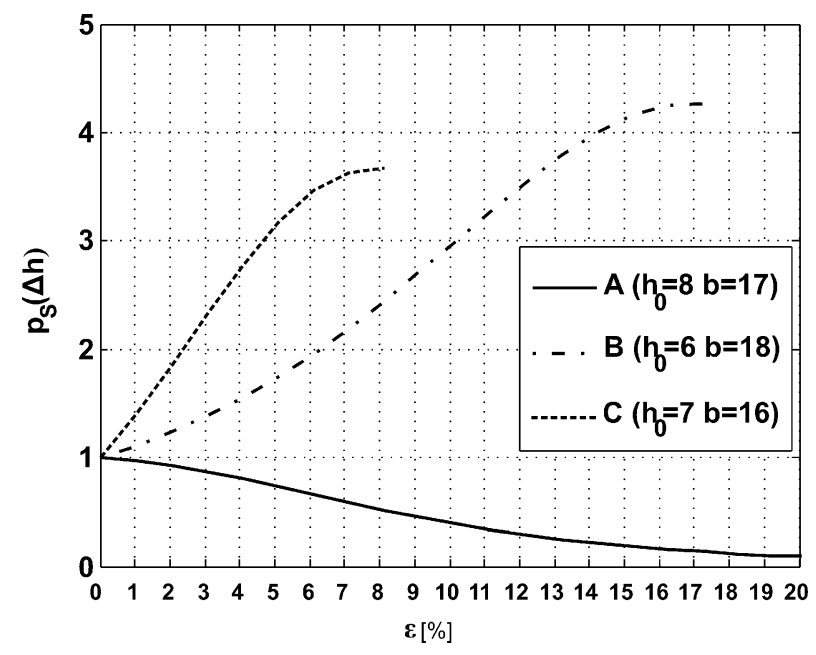

Fig. 4. Simulated inversion curves $p_{s}$ for three different realizations of the MLA radio-sensor, suited to monitor different strain dynamics.

TABLE I

DyNAMIC RANGE AND SENSITIVITY CORRESPONDING TO MLAS IN FIG. 4

\begin{tabular}{l|ccc} 
MLA & $\epsilon_{\max }[\%]$ & $p_{s}\left(\epsilon_{\max }\right)$ & $s[\%]$ \\
\hline $\mathrm{A}$ & 20 & 0.1 & -4.5 \\
$\mathrm{~B}$ & 17 & 4.3 & 19 \\
$\mathrm{C}$ & 7 & 3.7 & 39
\end{tabular}

$Z_{A}(\epsilon)$ (see again for instance Fig. 2), will be accordingly shifted like the resonance frequency of a dipole, that moves at lower of higher frequencies by changing the antenna length. In particular, a monotonically dumped response $\left(\epsilon_{\text {peak }} \rightarrow 0\right)$ is achieved by increasing $h_{0}$, e.g., the impedance curve $Z_{A}(\epsilon)$ is forced to move to the left.

The sensitivity $s$ (e.g., the amplitude of the peak of $p_{s}$ ) which is mainly fixed by the profile of $R_{A}$, as stated above, is instead dependent on both $\left(h_{0}, b\right)$ since the values of the resistance $R_{A}$ originates from the impedance's transformation ratio governed by the T-match [15], and ultimately by the parameter $b$.

For example, three different kinds of achievable sensing curves are represented in Fig. 4 for specific choices of the geometrical MLA parameters. The case A $\left(h_{0}=8 \mathrm{~mm}, b=17 \mathrm{~mm}\right)$ is fully monotonic $\left(\epsilon_{\text {peak }}=0\right)$ with negative slope (Table I) and it is hence suited to monitor smooth and wide deformations (for examples cracks over metallic structures and airplanes). Case B $\left(h_{0}=6 \mathrm{~mm}, b=18 \mathrm{~mm}\right)$ and case $\mathrm{C}\left(h_{0}=7 \mathrm{~mm}, b=16 \mathrm{~mm}\right)$ present instead two upward profiles with smaller ranges but greatly improved sensitivity as required for instance in SHM of civil buildings.

In real conditions, the design procedure requires to account for the environment surrounding the tag. The calculation of tag's gain and impedance has to include the presence of all the specific materials and structures wherein the strain sensor will be embedded, e.g., concrete or composite materials, each of them characterized in term of their electromagnetic and mechanical properties. This means that in order to extract absolute measurements about the experienced deformation by means of a specific MLA tag, data calibration should be performed by the help of more accurate measurements as well as by computer simulations on canonical environments.

\section{AN EXPERIMENTAL EXAMPLE}

A first $\mathrm{N}=1$ turn prototype of the radio strain sensor is fabricated (Fig. 5) by means of a folded copper wire $\left(r_{s}=0.5 \mathrm{~mm}\right)$ while the T-match section has been etched over a $15 \times 25 \times 0.96 \mathrm{~mm}$ FR4 substrate having single-side copper metallization. The copper wires are instead soldered on it. 


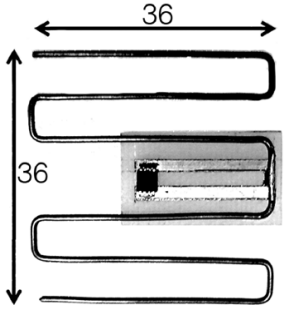

Fig. 5. Tag prototype. Size at rest in [mm]: $h_{0}=7, h_{1}=7, h=36, a=$ $4, b=19, w=36, r_{s}=0.5$.

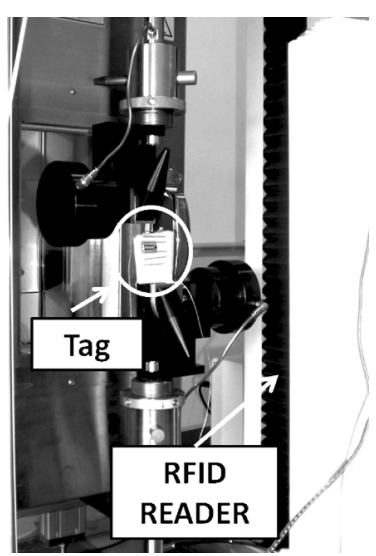

Fig. 6. Strain-gauge set-up connected to the MLA prototype by means of two plastic wires attached to the horizontal outer segments of the antenna.

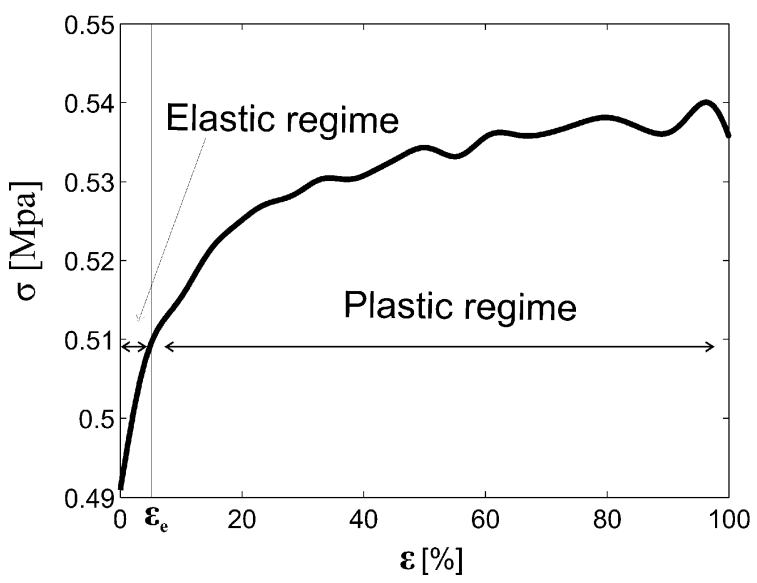

Fig. 7. Measured stress-strain curve of the MLA sensor prototype.

The geometrical parameters of the prototype are chosen in order to emphasize small deformations (Case B in Fig. 4), e.g., by acting on the upward-slope.

The tag is tested by means of a digital-controlled strain gauge (Fig. 6) which applies a 0-3 Newton axial tractive force to the antenna for a period of $80 \mathrm{~s}$. The MLA is fixed at the terminals of the strain gauge by means of two plastic wires attached to the horizontal outer segments of the antenna by means of loop knots.

During the test, a short range CAEN A528 reader connected to a planar inverted F-antenna (PIFA) with maximum $3.3 \mathrm{~dB}$ gain interrogates the tag by means of a fixed input power $P_{\text {in }}=20 \mathrm{dBm}$. The distance between reader and tag is chosen to $60 \mathrm{~cm}$ and the two antennas are oriented so that the tag is placed exactly in front of the reader's PIFA in polarization matching.

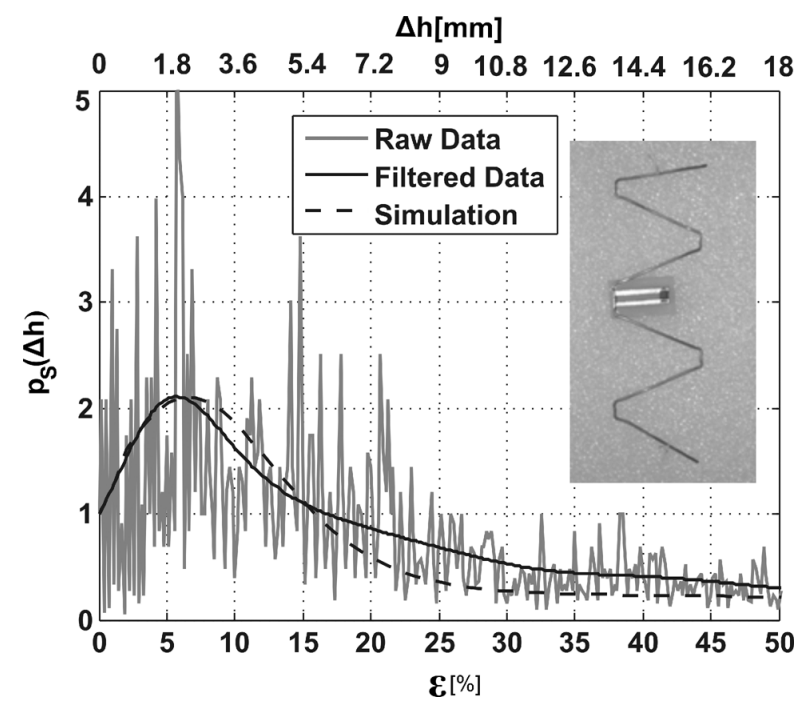

Fig. 8. Simulated and measured backscattered power as collected during the strain test. Data referred to the rest condition $\epsilon=0$ (unstrained tag).

The strain-stress $(\sigma)$ curve $^{1}$ relative to the tag's tractive test is shown in Fig. IV. Below the yield point, corresponding to a deformation $\epsilon_{e}=6 \%$, the tag will deform elastically (the stress is proportional to strain according to Hooke's law) and it will recover its original shape when the applied stress is removed. Once the yield point exceeds $\epsilon_{e}$, the deformation will become permanent and non-reversible (plastic regime) [9].

The measured backscattered power is visible in Fig. 8, superimposed to a low-pass filtering to smooth the typical oscillations of the received RFID signal. It is possible to observe a dynamic range $\epsilon_{\max } \equiv \epsilon_{\text {peak }}=$ $7 \%$, which covers the whole elastic deformation regime $(\epsilon=6 \%$ in Fig. 7), and a sensitivity $s=16 \%$. Just to have a numerical feeling, a $10 \%$ increase in the backscattered power indicates, from (9), an $\epsilon=$ $0.6 \%$ strain of the MLA, corresponding, through (7), to an elongation of approximately $\Delta h=216 \mu \mathrm{m}$. It is worth observing that, if a same layout was scaled to the RFID microwave frequency $\mathrm{f}=2450$ $\mathrm{MHz}$ (MLA sizes roughly reduced to one third), the elongation corresponding to $10 \%$ variation of backscattered power should be just $\Delta h=72 \mu \mathrm{m}$.

Finally, although the rough method used to connect the MLA to the tractive machine was rather imperfect, since it does not uniformly distribute the stress over the tag and produces a non exactly symmetric deformation (see the inset of Fig. 8), it is apparent that the experimental results are in reasonable agreements with mechanical and electrical simulations corroborating the idea to fully control the whole multiphysics response of the sensor.

\section{CONCLUSION}

Numerical analysis and early experimentations seem to encourage the use of meander line RFID tags as passive strain sensors. By acting on just two geometrical parameters of the sole impedance matching section it is possible to design several kinds of sensing responses suitable to large dynamic range of elongations as well as to very small phenomena.

The passive, low-cost (when compared with fully active devices) and pervasive nature of RFID technology can thus offer great advantages when traditional and powered solutions can not be feasible, e.g., when fixed infrastructures are not available, when the area to monitor is extremely wide or when the monitoring period is particularly long, months or years.

\footnotetext{
${ }^{1}$ The stress $\sigma$ is defined as the ratio between the applied tractive force and the cross-sectional area of action
} 
The shaped conducting wire forming the MLA may be directly embedded into the material to be observed, as in the case of concrete, with the purpose to achieve a kind of volumetric monitor, or it may be instead glued or sewn on an elastic substrate to be applied over a surface (two-dimensional monitoring). Alternatively, the emerging printing technologies could be used as in [7] to directly print the meander-line profile over the elastic support.

The recovery features of the sensor can be sensibly increased by employing super-elastic conductors, e.g., the shape-memory alloys (SMA) [17] already experimented to fabricate RFID tags [18]. Such conducting materials, for instance based on the Nickel-Titanium (Nitinol) alloy, can undergo large deformations at ambient temperature and then instantly revert back to their original shape (impressed during the fabrication process) when the stress is removed. Further investigations are however required to analyze the precision and the reproducibility of the Tag responses during cyclic loads.

Anyway, the bottleneck of this sensing platform is the resolution and stability of the RFID receiver onboard the reader. Actual commercial UHF reader may commonly offer a $0.8 \mathrm{~dB}$ resolution ( $8 \mathrm{bit}$ ) which means a $20 \%$ minimum appreciable variation in the detected RSSI signal corresponding to a minimum elongation of $0.43 \mathrm{~mm}$ in the case of the example prototype. Significantly better performances are hence expected with dedicated readers having a finer resolution in the analog to digital converter.

Finally, this class of sensors may be also used within a grid configuration, as conceived in [12], to achieve a detailed and vectorial monitoring of deformation over large surfaces.

\section{ACKNOWLEDGMENT}

The authors wish to thank prof. G. Costanza for his valuable support in performing experimental measurements.

\section{REFERENCES}

[1] G. Marrocco and F. Amato, "Self-sensing passive RFID: From theory to tag design and experimentation," presented at the 39th Eur. Microwave Conf., Rome, 2009.

[2] C. Occhiuzzi, G. Contri, and G. Marrocco, "RFID STENTag for passive vascular monitoring," presented at the EuCAP, Rome, Italy, Apr. 11-15, 2011.

[3] J. P. Lynch and K. J. Loh, "A summary review of wireless sensors and sensor networks for structural health monitoring," in Shock and Vibration Digest, Mar. 2006, vol. 38, no. 2, pp. 91-128.

[4] U. Tata, H. Huang, R. L. Carter, and J. C. Chiao, "Exploiting a patch antenna for strain measurements," Meas. Sci. Technol, vol. 20, 2009.

[5] A. Daliri, A. Galehdar, S. John, W. Rowe, and K. Ghorbani, "Circular microstrip patch antenna strain sensor for wireless structural health monitoring," in Proc. World Congress of Engineering, 2010.

[6] I. Mohammad and H. Huang, "Monitoring fatigue crack growth and opening using antenna sensors," Smart Mater. Struct., vol. 19, p. 055023,2010

[7] S. Merilampi, P. Ruuskanen, T. Björninen, L. Ukkonen, and L. Sydänheimo, "Printed passive UHF RFID tags as wearable strain sensors," presented at the 3rd Int. Symp. on Applied Sciences in Biomedical and Communication Technologies (ISABEL), Rome, 2010.

[8] G. Marrocco, "Gain-optimized self-resonant meander line antennas for RFID applications," IEEE Antennas Wireless Propag. Lett., vol. 2, p. 302305,2003

[9] R. C. Hibbeler, Engineering Mechanics: Combined Statics \& Dynamics, 12th ed. Englewood Cliffs, NJ: Prentice Hall, 2010.

[10] P. V. Nikitin and K. V. S. Rao, "Theory and measurement of backscattering from RFID tags," IEEE Antennas Propag. Mag., vol. 48, no. 6, pp. 212-218, Dec. 2006.

[11] F. Fuschini, C. Piersanti, F. Paolazzi, and G. Falciasecca, "Analytical approach to the backscattering from UHF-RFID transponder," IEEE Antennas Wireless Propag. Lett., vol. 7, pp. 33-35, 2008.
[12] G. Marrocco, "RFID GRIDS: Part I-electromagnetic theory," IEEE Trans. Antennas Propag., vol. 59, no. 3, pp. 1010-1026, Mar. 2011.

[13] A. Taflove and S. C. Hagness, Computational Electrodynamics: The Finite-Difference Time-Domain Method, 3rd ed. Norwood, MA: Artech House, 2005.

[14] S. Best, "On the resonant properties of the Koch fractal and other wire monopole antennas," IEEE Trans. Antennas Wireless Propag. Lett., vol. 1, pp. 74-76, 2002.

[15] C. Balanis, Antenna Theory: Analysis and Design, 2nd ed. New York: Wiley, 1997, pp. 472-475.

[16] G. Marrocco, "The art of UHF RFID antenna design: Impedancematching and size-reduction techniques," IEEE Antennas Propag. Mag., vol. 50, no. 1, pp. 66-96, Feb. 2008.

[17] H. Funakubo, Shape Memory Alloys. New York: Gordon and Breach, 1987.

[18] S. Caizzone, C. Occhiuzzi, and G. Marrocco, "Multi-chip RFID antenna integrating shape-memory alloys for detection of thermal thresholds," IEEE Trans. Antennas Propag., vol. 59, pp. 2488-2494, Jul. 2011.

\section{Millimeter-Wave Miniaturized Substrate Integrated Multibeam Antenna}

Yu Jian Cheng and Yong Fan

\begin{abstract}
Substrate integrated waveguide (SIW) presents an excellent candidate in the development of millimeter-wave integrated printed multibeam antenna depending on its inherent advantages. But the SIW-based multibeam antenna usually occupies a large circuit size. To overcome this weakness, we propose a novel miniaturized SIW multibeam antenna at the center frequency $33.5 \mathrm{GHz}$. Such a structure can be considered as an asymmetric multimode beamforming network (BFN) directly integrated with a compact antipodal linearly tapered slot antenna (ALTSA) array without additional phase shifters or connection lines. It has smaller circuit size compared with the state-of-the-art SIW multibeam antenna technology. Four beams with different beam directions can be generated to cover the angle range of around $76^{\circ}$ with $3 \mathrm{~dB}$ beam-widths at the center frequency.
\end{abstract}

Index Terms-Miniaturization, multibeam antenna, multimode, substrate integrated waveguide (SIW).

\section{INTRODUCTION}

Multibeam antenna is considered to be one of the most effective method to increase capacity and reduce power consumption. Thus it has been widely used in smart communication system [1]. Besides, the capability of rapid and accurate beam scanning permits the multibeam antenna to be applied in many other fields, such as electronically-steered radar [2].

As a very promising technology, substrate integrated waveguide (SIW) [3]-[6] scheme combines the excellent features of both planar

Manuscript received December 02, 2010; revised June 14, 2011; accepted June 16, 2011. Date of publication August 22, 2011; date of current version December 02,2011 . This work is supported in part by the National Natural Science Foundation of China (NSFC) under Grant 61001028, in part by Research Fund for the Doctoral Program of Higher Education of China (RFDP) under Grant 20100185110014, and in part by the Fundamental Research Funds for the Central Universities under Grant ZYGX2010J019.

The authors are with the EHF Key Lab of Fundamental Science, School of Electronic Engineering, University of Electronic Science and Technology of China (UESTC), Chengdu 611731, China (e-mail: yjcheng@em field.org).

Color versions of one or more of the figures in this communication are available online at http://ieeexplore.ieee.org.

Digital Object Identifier 10.1109/TAP.2011.2165497 